\title{
Suggestions on Effective Organization, Mobilization and Management of Non-Governmental Voluntary Organizations from the Practice of the Prevention and Control of the Pandemic
}

\author{
Wenying Li \\ Ningbo University of Technology, Ningbo, China \\ Email: lwy@nbut.edu.cn
}

How to cite this paper: Li, W. Y. (2020). Suggestions on Effective Organization, Mobilization and Management of Non-Governmental Voluntary Organizations from the Practice of the Prevention and Control of the Pandemic. Advances in Applied Sociology, 10, 525-530.

https://doi.org/10.4236/aasoci.2020.1012032

Received: December 15, 2020

Accepted: December 27, 2020

Published: December 30, 2020

Copyright $\odot 2020$ by author(s) and Scientific Research Publishing Inc. This work is licensed under the Creative Commons Attribution International License (CC BY 4.0).

http://creativecommons.org/licenses/by/4.0/

(c) (i) Open Access

\begin{abstract}
Voluntary service plays an important part in the united fight against the pandemic, which is an essential symbol reflecting the progress of social civilization. Since the reform and opening-up, more freedom has been given in the development of economy, culture and other social resources, and the government's function is changing from "government-based" to "individual-based". Citizens are more aware of their role in the society, and the community-level governance system is steadily improved. However, a large number of public affairs and social problems have arisen, which are difficult to be addressed by the government alone. New social strengths apart from the government are urgently needed to effectively to give support and assistance. They fundamentally provide conditions for the development of non-govern- mental volunteer organizations.
\end{abstract}

\section{Keywords}

Suggestions, Non-Governmental Voluntary Organizations, Management

\section{Current Situation}

Currently, the city of Wuhan in Hubei province is combating Covid-19 with all walks of life involved. To save this city, medical staffs from all around the country are sent to work at the frontline. Voluntary jobs are done in form of professional medical screening, temperature measurement, epidemic prevention, social 
village propaganda, road patrolling and mask-production assistance. There are also volunteers who help harvest agricultural products, buy foods for those in need and take care of special groups including the old and the disabled. As of March 28, there were 161 million real-name volunteers working on totally 3.5317 million volunteer projects, pushing voluntary hours to 1.962 billion. Among them, Zhejiang province provided 9.486500 real-name volunteers (Ningbo City sent $2,144,707$ real-name volunteers, accounting for $22.61 \%$ of the province, among which 93,316 were first-line volunteers). Correspondingly, the service hours of Zhejiang province hit 6.163900 , ranking the sixth among the 34 provincial-level administrative regions in China, after Henan with 12.157 million, Shandong with 11.781 million, Guangdong with 10.37 million, Jiangsu with 10.376 million, and Anhui with 9.8684 million. Combating in the first line and behaving remarkably, these volunteers full of devotion spirit are spontaneously forming precious social organizations. It is these organizations that effectively prevented the spread of the epidemic, saved government image damage resulting from some decision-making errors and improper measures cause bony brand effect in the process of dealing with this security crisis. Unlike the top-down administrative governance system of government agencies, these non-governmental volunteer organizations do not cling to administrative instructions, regardless of in volunteer's recruitment and dispatching, material preparation, or organization operation. Instead, rooted at organizations of social communities, these organizations operate entirely independently. Besides, what is noteworthy is that these organizations distinguish from corporate organizations as well, evidenced by that these organizations, formed spontaneously by citizens, are endowed with non-profit and volunteering nature. Volunteers and private voluntary organizations are known for quick reactions, wide distribution and fast operations, exactly remedy the deficiency of the role that the government plays in this outbreak. The pattern of online and off-line service by them also won the hearts of a vast number of people. Yet in the midst of voluntary epidemic prevention and control, some problems are also exposed, leading to failure in volunteering, such as delay for curing or secondary damage by improper methods (Fang, 2018).

\section{Remaining Problems}

\subsection{Lack of Legal Protection of Laws and Regulations}

At present, there are a total of 18 domestic legislation concerning the incidence and emergency management and more than 200 administrative regulations such as The Measures for The Administration of The Emergency Contingency Plans and Regulations on The Public Health Emergencies. And Regulations formulated by commissions and local governments are countless. But all these laws and regulations share a characteristic, that is, overemphasis on the management of government authority and the lack of social mobilization strength. For example, article 11 of the first chapter of the Emergency Response Law of the People's Republic of China stipulates that "citizens, legal persons and other organizations 
have the obligation to participate in the response to emergencies." But in what ways? Who organizes the leadership? At what stage of the process do you participate in the emergency? What are the procedures involved? The details await explanation and specification attaching to a complete set of document. For example, Opinions of The State Council on Comprehensively Strengthening the Emergency Management Work stipulated that we should study and formulate the methods to mobilize and encourage volunteers to participate in the emergency rescue work, and to strengthen the recruitment, organization and training of the volunteer team. But what measures should be taken to encourage voluntary organizations to participate in the emergency rescue work? Which department should take the lead in volunteer recruitment, organization, and training? What is the source fund? These problems lack further explanation. The role of the government in the volunteer service is not stipulated in the law, nor do the protective specifications on the rights of enterprise. All these lead to the fact that cooperates have less obvious desires for funding voluntary activities. The lack of laws and regulations unable voluntary organizations to get involved in emergency management. All insufficiency in the macro-design and management mechanism results in a breakdown in volunteer emergency system (Jia, 2020).

\subsection{An Impediment in the Operation Mechanism of Voluntary Service}

Firstly, there is a lack of unified leadership in the Command Center. Our audit registration management is only limited in civil affairs departments. Unified volunteer management institutions are not available in most places, limited in jurisdictions. The scarcity of unified planning, supervision and guidance, volunteer services are mostly stay in superficial level, devoid of a long-term goal and predictability, let alone an overall coordination. Some local governments have established emergency rescue organizations, but there remain a number of problems, like ineffective management of emergency teams, personnel, allocation of rescue equipment and maintenance of response mechanism. A sound technical support system is ill-established, making numerous information errors, resulting in the waste of resources and the inefficiency of volunteer service. For example, on the home page of "Ningbo Volunteer Service" website, "Emergency Relief Type", the Epidemic Community Volunteer Project of Hongtang Community, Jiangbei District planed to recruit 240 people, but only 1 person was employed in the end. Dongying New Village, Neighborhood Committee of Dongying Community, Panhuo Street in Yinzhou District, planed to recruit 50 people, but no one was employed (as of March 28). Secondly, the recruitment, business training, deployment management, logistics support and incentive of volunteer organizations are still on the superficial level but are not targeted at individual volunteers, which results in a lack of specific management system for the training content of volunteers. Third, there is a general shortage of funds, basic material security of voluntary services and personal health and safety. 


\subsection{Scattered Volunteer Organizations}

At present, non-governmental volunteer organizations have dispersed their emergency rescue forces, and established relatively independent emergency countermeasures within their own organizations according to the characteristics of their own disasters. Each is in its own array without interfering with others. This directly results in the command and coordination which is limited to a narrow field and unable to coordinate the overall unified command. During this outbreak, Wuhan severely lacked medical personnel and medical resources Meanwhile there is no enough room for patients to live in hospital and nurses wore diapers and can't drink water. Just relying on Hubei province health systems of emergency power and resources, was obviously insufficient. Regarding emergency rescue force of temporary organization, such as the provinces rescue of medical personnel, folk spontaneous volunteers and the army, there is something wrong with responsibility, mechanism, thus difficult to together, the whole rescue ability into full play (Wang, 2018).

\section{Suggestions}

\subsection{Coordination between Strong Center and De-Center}

We should give play to the central role led by the government, avoid loose organization, breakdown and shirking responsibility. We should make it clear that "whoever recruits and organizes volunteers is in charge of security", which will be conducive to improving rescue efficiency. Emphasis should also be laid on de-center. Flexible and mobile volunteer organizations tend to organize large-scale rescue forces at the beginning of the disasters. Volunteers and the flexible and loose organizational forms of voluntary organizations are easy to carry out at the grassroots level, giving full play to the role of science popularization, risk monitoring and other functions that are difficult for the government to achieve. Therefore, we should coordinate the strong center and de-center, give consideration to both the upward and downward movement of the center.

\subsection{Build a Common Standardization System for Individual Volunteers with Both Professionalism and Routineness}

It is suggested to refer to the grading assessment method of THE United Nations rescue team. At the same time, the general standards for individual volunteers are formulated based on the categories and contents of voluntary services, volunteer education background (educational background), training duration, working years, skill assessment and necessary knowledge. First of all, volunteers are divided into four levels according to their functions in voluntary activities: primary, intermediate, senior and super. According to the process of emergency handling before, during and after, junior volunteers are responsible for basic handling work, such as table data statistics. Intermediate volunteers are responsible for classification, evacuation and basic rescue, fully assist professional rescue; Senior volunteers must be professional volunteers with strong professional 
skills required for emergencies, and be responsible for the coordination and scheduling of all on-site rescue volunteers; In addition to the overall command of rescue operations, special volunteers should also be responsible for the recruitment, training and guidance of primary, secondary and senior volunteers during the period of non-voluntary service, as well as the work of various civil voluntary organizations. They are management volunteers. The general standard can also be regarded as a promotion system for volunteers, which is similar to the professional title evaluation of university teachers. After a certain number of years, junior university lecturers will apply for the senior title after completing the corresponding education hours and publishing relevant scientific research papers within the tenure. Similarly, the general standard for individual volunteers is that each level has a corresponding training period. According to the different educational background, the corresponding working years will be promoted to the senior level after the assessment. According to the severity of the emergency, volunteers of the corresponding level shall be assigned according to the degree of the incident or the order of priority of the degree (Zhao \& Mu, 2020).

\subsection{Rights and Interests Protection and Social Incentives}

Private voluntary organizations, though not for any material reward, not driven by private interests, in order to improve social services, promote social development, voluntarily contribute their personal time and spirit, but this does not mean that there is no need to develop corresponding incentive system and protection system. Civil voluntary organizations are encouraged to participate in emergency management while protecting their rights and interests. First, tax incentives and credit incentives. In major outbreaks or sudden disaster, enterprises or individuals who provide volunteer services such as financial resources, material products, especially those with huge amounts and outstanding contributions, should be commended and exempted or given preferential treatment in terms of taxation. Record the volunteer service time into the social credit system and provide incentives and feedback on housing purchases, insurance, loans, etc. Second, encourage people to choose careers and start their own businesses. Encourage government offices, enterprises and public institutions to incorporate voluntary services into the recruitment inspection when setting recruitment conditions; Encourage companies and enterprises to give priority to recruiting civilian volunteers with good records of volunteer service under the same conditions; In the evaluation and assessment of party membership, priority should be given to volunteers with a certain length of time of volunteer service, so that civil volunteers can get spiritual encouragement, so that volunteers who contribute love and help others can feel the affirmation and support from the national government and all sectors of society. In this way, they are more willing to do a good job and do good deeds.

\section{Conflicts of Interest}

The author declares no conflicts of interest regarding the publication of this paper. 


\section{References}

Fang W. (2018). Voluntary and Professional Choices of Non-Governmental Organizations: A Case Study of a Child Autism Rehabilitation Institution in Hangzhou. Social Sciences, 3, 73-81.

Jia, X. J. (2020). “Coordination" or "Information": The Collaborative Model of Government and Voluntary Mechanisms in the Fight against the Epidemic. China Non-profit Review, 25, 8-12.

Wang, T. (2018). A Brief Talk on Voluntary Failure of Non-Governmental Organizations in my country. China Collective Economy, 30, 72-73.

Zhao, C. G., \& Mu, T. (2020). An Analysis of the Predicament of the Integration of Community Education into Social Governance from the Perspective of Non-Governmental Organization Participation. Frontier Economy and Culture, 7, 75-76. 